KYUNGPOOK Math. J. 54(2014), 349-363

http://dx.doi.org/10.5666/KMJ.2014.54.3.349

\title{
Uniqueness of Entire Functions and Differential Polynomials Sharing one Value
}

\author{
XiAO-Bin ZhanG* \\ College of Science, Civil Aviation University of China, Tianjin 300300, China \\ e-mail : xbzhang1016@mail.sdu.edu.cn \\ DA-WeI MENG \\ College of mathematics and statistics, Xidian University, Xi'an, Shaanxi 710126 \\ China \\ e-mail : goths511@163.com
}

ABSTRACT. In this paper, we shall utilize Nevanlinna value distribution theory to study the uniqueness problems on entire functions and differential polynomials sharing one value. Our theorems improve or generalize some results of Zhang and Lin, Chen, Zhang, Lin and Chen.

\section{Introduction and Main Results}

Let $\mathbb{C}$ denote the complex plane and $f(z)$ be a non-constant meromorphic function on $\mathbb{C}$. We assume the reader is familiar with the standard notion used in the Nevanlinna value distribution theory such as the characteristic function $T(r, f)$, the proximate function $m(r, f)$, the counting function $N(r, f)$ and $S(r, f)$ denotes any quantity that satisfies the condition $S(r, f)=o(T(r, f))$ as $r \rightarrow \infty$ outside of a possible exceptional set of finite linear measure.

Let $f(z)$ and $g(z)$ be two non-constant meromorphic functions. Let $a$ be a finite complex number. We say that $f(z), g(z)$ share the value $a$ CM (counting multiplicities) if $f(z), g(z)$ have the same a-points with the same multiplicities and we say that $f(z), g(z)$ share the value $a$ IM (ignoring multiplicities) if we do not consider the multiplicities. We denote by $E_{m)}(a ; f)$ the set of all $a$ - points of $f$ with multiplicities not greater than $m$, where an $a$ - point is counted according to its multiplicity. If for $a \in \mathbb{C}, E_{\infty)}(a ; f)=E_{\infty}(a ; g)$, it means that $f, g$ share the value $a \mathrm{CM}$. We denote by $N_{k}\left(r, \frac{1}{f-a}\right)$ (or $\left.\bar{N}_{k}\left(r, \frac{1}{f-a}\right)\right)$ the counting function for zeros of $f-a$ with multiplicity $\leq k$ (ignoring multiplicities), and by $N_{(k}\left(r, \frac{1}{f-a}\right)$

\section{* Corresponding Author.}

Received March 16, 2011; accepted January 28, 2014.

2010 Mathematics Subject Classification: 30D35.

Key words and phrases: uniqueness, entire function, differential polynomial, sharing value. 
(or $\bar{N}_{(k}\left(r, \frac{1}{f-a}\right)$ ) the counting function for zeros of $f-a$ with multiplicity $\geq k$ (ignoring multiplicities). Moreover we set $N_{k}\left(r, \frac{1}{f-a}\right)=\bar{N}\left(r, \frac{1}{f-a}\right)+\bar{N}_{(2}\left(r, \frac{1}{f-a}\right)+$ $\bar{N}_{(3}\left(r, \frac{1}{f-a}\right)+\cdots+\bar{N}_{(k}\left(r, \frac{1}{f-a}\right)$.

For the sake of simplicity we also use the notion $m^{*}:=\chi_{\mu} m$, where $\chi_{\mu}=\left\{\begin{array}{c}0, \mu=0 \\ 1, \mu \neq 0\end{array}\right.$

Recently, corresponding to a famous question of Hayman [5], Fang and Hua [4], Yang and Hua [11] obtained the following result.

Theorem A: Let $f$ and $g$ be two non-constant entire functions, $n \geq 6$ be a positive integer. If $f^{n}(z) f^{\prime}(z)$ and $g^{n}(z) g^{\prime}(z)$ share $1 C M$, then either $f(z)=c_{1} e^{c z}, g(z)=$ $c_{2} e^{-c z}$, where $c_{1}, c_{2}$ and $c$ are three constants satisfying $\left(c_{1} c_{2}\right)^{n+1} c^{2}=-1$, or $f(z) \equiv \operatorname{tg}(z)$ for a constant $t$ such that $t^{n+1}=1$.

Note that $f^{n}(z) f^{\prime}(z)=\frac{1}{n+1}\left(f^{n+1}(z)\right)^{\prime}$. Fang [3] considered $k$-th derivative instead of 1-th derivative and proved the following theorems.

Theorem B: Let $f$ and $g$ be two non-constant entire functions, and let $n, k$ be two positive integers with $n>2 k+4$. If $\left(f^{n}(z)\right)^{(k)}$ and $\left(g^{n}(z)\right)^{(k)}$ share $1 C M$, then either $f(z)=c_{1} e^{c z}, g(z)=c_{2} e^{-c z}$, where $c_{1}, c_{2}$ and $c$ are three constants satisfying $(-1)^{k}\left(c_{1} c_{2}\right)^{n}(n c)^{2 k}=1$, or $f(z) \equiv \operatorname{tg}(z)$ for a constant $t$ such that $t^{n}=1$.

Theorem C: Let $f$ and $g$ be two non-constant entire functions, and let $n, k$ be two positive integers with $n>2 k+8$. If $\left(f^{n}(z)(f(z)-1)\right)^{(k)}$ and $\left(g^{n}(z)(g(z)-1)\right)^{(k)}$ share $1 C M$, then $f(z) \equiv g(z)$.

Chen, Zhang, Lin and Chen [2] improved Theorems B and C and proved the following theorems.

Theorem D: Let $f$ and $g$ be two non-constant entire functions, and let $n, k$ and $m$ be three positive integers. If $E_{m)}\left(1,\left(f^{n}\right)^{(k)}\right)=E_{m)}\left(1,\left(g^{n}\right)^{(k)}\right)$ and

(i) if $m=1$ and $n>4 k+6$, then either $f(z)=c_{1} e^{c z}, g(z)=c_{2} e^{-c z}$, where $c_{1}, c_{2}$ and $c$ are three constants satisfying $(-1)^{k}\left(c_{1} c_{2}\right)^{n}(n c)^{2 k}=1$, or $f(z) \equiv \operatorname{tg}(z)$ for a constant $t$ such that $t^{n}=1$; or

(ii) if $m=2$ and $n>\frac{5 k+9}{2}$, then either $f(z)=c_{1} e^{c z}, g(z)=c_{2} e^{-c z}$, where $c_{1}, c_{2}$ and $c$ are three constants satisfying $(-1)^{k}\left(c_{1} c_{2}\right)^{n}(n c)^{2 k}=1$, or $f(z) \equiv \operatorname{tg}(z)$ for a constant $t$ such that $t^{n}=1$; or

(iii) if $m=3$ and $n>2 k+4$, then either $f(z)=c_{1} e^{c z}, g(z)=c_{2} e^{-c z}$, where $c_{1}, c_{2}$ and $c$ are three constants satisfying $(-1)^{k}\left(c_{1} c_{2}\right)^{n}(n c)^{2 k}=1$, or $f(z) \equiv \operatorname{tg}(z)$ for a constant $t$ such that $t^{n}=1$.

Theorem E: Let $f$ and $g$ be two non-constant entire functions, and let $n, k$ and $m$ be three positive integers. If $E_{m)}\left(1,\left(f^{n}(f-1)\right)^{(k)}\right)=E_{m)}\left(1,\left(g^{n}(g-1)\right)^{(k)}\right)$ and 
(i) if $m=1$ and $n>4 k+11$, then $f(z) \equiv g(z)$; or

(ii) if $m=2$ and $n>\frac{5 k+16}{2}$, then $f(z) \equiv g(z)$; or

(iii) if $m=3$ and $n>2 k+7$, then $f(z) \equiv g(z)$.

Moreover, Zhang and Lin [13] considered some general differential polynomials such as $\left(f^{n}(z)\left(f^{m}(z)-1\right)\right)^{(k)}$ and $\left(f^{n}(z)(f(z)-1)^{m}\right)^{(k)}$. They proved the following theorems.

Theorem F: Let $f$ and $g$ be two non-constant entire functions, and let $n, m$ and $k$ be three positive integers with $n>2 k+m^{*}+4$, and $\lambda, \mu$ be constants such that $|\lambda|+|\mu| \neq 0$. If $\left(f^{n}(z)\left(\mu f^{m}(z)+\lambda\right)\right)^{(k)}$ and $\left(g^{n}(z)\left(\mu g^{m}(z)+\lambda\right)\right)^{(k)}$ share $1 C M$, then

(i) when $\lambda \mu \neq 0, f \equiv t g$, for a constant $t$ such that $t^{n}=1$ and $t^{m}=1$;

(ii) when $\lambda \mu=0$, either $f(z) \equiv t g(z)$, where $t$ is a constant satisfying $t^{n+m^{*}}=$ 1 , or $f(z)=c_{1} e^{c z}, g(z)=c_{2} e^{-c z}$, where $c_{1}, c_{2}$ and $c$ are three constants satisfying $(-1)^{k} \lambda^{2}\left(c_{1} c_{2}\right)^{n+m^{*}}\left[\left(n+m^{*}\right) c\right]^{2 k}=1$ or $(-1)^{k} \mu^{2}\left(c_{1} c_{2}\right)^{n+m^{*}}[(n+$ $\left.\left.m^{*}\right) c\right]^{2 k}=1$.

Theorem G: Let $f$ and $g$ be two non-constant entire functions, and let $n, m, k$ be three positive integers with $n>2 k+m+4$. If $\left(f^{n}(z)(f(z)-1)^{m}\right)^{(k)}$ and $\left(g^{n}(z)(g(z)-\right.$ $\left.1)^{m}\right)^{(k)}$ share $1 C M$, then either $f(z) \equiv g(z)$, or $f$ and $g$ satisfy the algebraic equation $R(f, g) \equiv 0$, where $R\left(\omega_{1}, \omega_{2}\right)=\omega_{1}^{n}\left(\omega_{1}-1\right)^{m}-\omega_{2}^{n}\left(\omega_{2}-1\right)^{m}$.

Using the idea of weighted sharing, Liu [6] improved and generalized Theorem $\mathrm{F}$ by proving the following result.

Theorem H: Let $f$ and $g$ be two non-constant meromorphic functions, and let $n$, $m$ and $k$ be three positive integers, and $\lambda, \mu$ be two constants such that $|\lambda|+|\mu| \neq 0$. If $E_{l}\left(1,\left[f^{n}(z)\left(\mu f^{m}(z)+\lambda\right)\right]^{(k)}\right)=E_{l}\left(1,\left[g^{n}(z)\left(\mu g^{m}(z)+\lambda\right)\right]^{(k)}\right)$, and one of the following conditions holds,

(1) $l \geq 2$ and $n \geq 3 m^{*}+3 k+8$;

(2) $l=1$ and $n \geq 4 m^{*}+5 k+10$;

(3) $l=0$ and $n \geq 6 m^{*}+9 k+14$.

Then (i) when $\lambda \mu \neq 0$, if $m \geq 2$ and $\delta(\infty, f)>\frac{3}{n+m}$, then $f \equiv g$; If $m=1$ and $\delta(\infty, f)>\frac{3}{n+1}$, then $f \equiv g$;

(ii) when $\lambda \mu=0$, if $f \neq \infty$ and $g \neq \infty$, then either $f(z) \equiv \operatorname{tg}(z)$, where $t$ is a constant satisfying $t^{n+m^{*}}=1$, or $f(z)=c_{1} e^{c z}, g(z)=c_{2} e^{-c z}$, where $c_{1}$, $c_{2}$ and $c$ are three constants satisfying $(-1)^{k} \lambda^{2}\left(c_{1} c_{2}\right)^{n+m^{*}}\left[\left(n+m^{*}\right) c\right]^{2 k}=1$ or $(-1)^{k} \mu^{2}\left(c_{1} c_{2}\right)^{n+m^{*}}\left[\left(n+m^{*}\right) c\right]^{2 k}=1$.

For more related results, see [1,7-9]. 
Take notice of the conditions "if $f \neq \infty$ and $g \neq \infty$ " in (ii) of Theorem $\mathrm{H}$, actually $f$ and $g$ are entire functions under the conditions. In this paper, we improve Theorem F and G in another way. Now we state our main results of the paper.

Theorem 1.1. Let $f$ and $g$ be two non-constant entire functions, let $n, m$ and $k$ be three positive integers with $n>2 k+m^{*}+4$, and $\lambda, \mu$ be constants such that $|\lambda|+|\mu| \neq 0$. If $E_{3)}\left(1 ;\left(f^{n}(z)\left(\mu f^{m}(z)+\lambda\right)\right)^{(k)}\right)=E_{3)}\left(1 ;\left(g^{n}(z)\left(\mu g^{m}(z)+\lambda\right)\right)^{(k)}\right)$, then the conclusions of the Theorem $F$ hold.

Theorem 1.2. Let $f$ and $g$ be two non-constant entire functions, let $n, m$ and $k$ be three positive integers with $n>\frac{5 k+3 m^{*}+9}{2}$, and $\lambda, \mu$ be constants such that $|\lambda|+|\mu| \neq 0$. If $E_{2)}\left(1 ;\left(f^{n}(z)\left(\mu f^{m}(z)+\lambda\right)^{(k)}\right)=E_{2)}\left(1 ;\left(g^{n}(z)\left(\mu g^{m}(z)+\lambda\right)\right)^{(k)}\right)\right.$, then the conclusions of the Theorem $F$ hold.

Theorem 1.3. Let $f$ and $g$ be two non-constant entire functions, let $n, m$ and $k$ be three positive integers with $n>4 k+3 m^{*}+6$, and $\lambda, \mu$ be constants such that $|\lambda|+|\mu| \neq 0$. If $E_{1)}\left(1 ;\left(f^{n}(z)\left(\mu f^{m}(z)+\lambda\right)\right)^{(k)}\right)=E_{1)}\left(1 ;\left(g^{n}(z)\left(\mu g^{m}(z)+\lambda\right)\right)^{(k)}\right)$, then the conclusions of the Theorem $F$ hold.

Theorem 1.4. Let $f$ and $g$ be two non-constant entire functions, and let $n, m, k$ be three positive integers with $n>2 k+m+4$. If $E_{3)}\left(1 ;\left(f^{n}(z)(f(z)-1)^{m}\right)^{(k)}\right)=$ $E_{3)}\left(1 ;\left(g^{n}(z)(g(z)-1)^{m}\right)^{(k)}\right)$, then the conclusions of the Theorem $G$ hold.

Theorem 1.5. Let $f$ and $g$ be two non-constant entire functions, and let $n, m, k$ be three positive integers with $n>\frac{5 k+2 m+9+\min \{m, k+1\}}{2}$. If $E_{2)}\left(1 ;\left(f^{n}(z)(f(z)-\right.\right.$ $\left.\left.1)^{m}\right)^{(k)}\right)=E_{2)}\left(1 ;\left(g^{n}(z)(g(z)-1)^{m}\right)^{(k)}\right)$, then the conclusions of the Theorem $G$ hold.

Theorem 1.6. Let $f$ and $g$ be two non-constant entire functions, and let $n, m, k$ be three positive integers with $n>4 k+m+6+2 \min \{m, k+1\}$. If $E_{1)}\left(1 ;\left(f^{n}(z)(f(z)-\right.\right.$ $\left.\left.1)^{m}\right)^{(k)}\right)=E_{1)}\left(1 ;\left(g^{n}(z)(g(z)-1)^{m}\right)^{(k)}\right)$, then the conclusions of the Theorem $G$ hold.

Remark 1.1. Theorem 1.1 improves Theorem F, Theorem 1.4 improves Theorem G.

Remark 1.2. Let $\mu=0$ and $\lambda=1$ in Theorems 1.1, 1.2 and 1.3, the three theorems also generalize Theorem D.

Remark 1.3. Let $m=1$ in Theorem 1.4, 1.5 and 1.6, the three theorems also improve and generalize Theorem E. In fact, by Theorem G, if $f \not \equiv g$, then $f^{n}(f-$ $1)=g^{n}(g-1)$. Let $h=\frac{f}{g}$, we have $g=\frac{h^{n}-1}{h^{n+1}-1}=\frac{(h-u)\left(h-u^{2}\right) \cdots\left(h-u^{n-1}\right)}{(h-v)\left(h-v^{2}\right) \cdots\left(h-v^{n}\right)}$, where $u=\exp (2 \pi i / n)$ and $v=\exp (2 \pi i /(n+1))$. Since $g$ is entire and $h$ is a non-constant meromorphic function, $v^{j}(1 \leq j \leq n)$ are Picard exceptional values of $h$. This is impossible. Therefore $f \equiv g$. 


\section{Some Lemmas}

Lemma $2.1([3])$. Let $f(z)$ be a non-constant meromorphic function and let $a_{0}, a_{1}, \cdots a_{n}(\neq 0)$ be finite complex numbers. Then

$$
T\left(r, a_{n} f^{n}+a_{n-1} f^{n-1}+\cdots+a_{0}\right)=n T(r, f)+S(r, f) .
$$

Lemma $2.2([10])$. Let $f(z)$ be a non-constant meromorphic function, and let $k$ be a positive integer. Suppose that $f^{(k)} \not \equiv 0$, then

$$
N\left(r, \frac{1}{f^{(k)}}\right) \leq N\left(r, \frac{1}{f}\right)+k \bar{N}(r, f)+S(r, f) .
$$

Lemma 2.3 ([12]). Let $f(z)$ be a non-constant meromorphic function, $s, k$ be two positive integers. Then

$$
\begin{gathered}
N_{s}\left(r, \frac{1}{f^{(k)}}\right) \leq T\left(r, f^{(k)}\right)-T(r, f)+N_{s+k}\left(r, \frac{1}{f}\right)+S(r, f), \\
N_{s}\left(r, \frac{1}{f^{(k)}}\right) \leq k \bar{N}(r, f)+N_{s+k}\left(r, \frac{1}{f}\right)+S(r, f) .
\end{gathered}
$$

Lemma 2.4. Let $f(z)$ and $g(z)$ be two non-constant entire functions, let $n, m$, and $k$ be three positive integers with $n>k+2$ and $\lambda, \mu$ be constants such that $|\lambda|+|\mu| \neq 0$. Set

$$
\begin{gathered}
F=\left(f^{n}(z)\left(\mu f^{m}(z)+\lambda\right)\right)^{(k)}, \quad G=\left(g^{n}(z)\left(\mu g^{m}(z)+\lambda\right)\right)^{(k)}, \\
F^{*}=f^{n}(z)\left(\mu f^{m}(z)+\lambda\right), \quad G^{*}=g^{n}(z)\left(\mu g^{m}(z)+\lambda\right) . \\
H=\left[\frac{F^{\prime \prime}}{F^{\prime}}-\frac{2 F^{\prime}}{F-1}\right]-\left[\frac{G^{\prime \prime}}{G^{\prime}}-\frac{2 G^{\prime}}{G-1}\right] .
\end{gathered}
$$

If $E_{3)}(1 ; F)=E_{3)}(1 ; G)$ and $H \not \equiv 0$, then

$$
\begin{aligned}
m\left(r, \frac{1}{F}\right)+m\left(r, \frac{1}{G}\right) \leq & N\left(r, \frac{1}{F}\right)+N\left(r, \frac{1}{G}\right)-2(n-k-2) N\left(r, \frac{1}{f}\right) \\
& -2(n-k-2) N\left(r, \frac{1}{g}\right)+S(r, f)+S(r, g) .
\end{aligned}
$$

Proof. Since $E_{3)}(1 ; F)=E_{3)}(1 ; G)$, a simple computation shows that $H\left(z_{0}\right)=0$ if $z_{0}$ is a simple zero of $F-1$ and $G-1$.

As $H \not \equiv 0$, by the lemma of logarithmic derivatives, we get $m(r, H)=S(r, f)+$ $S(r, g)$, so

$$
\begin{aligned}
N_{1)}\left(r, \frac{1}{F-1}\right) & =N_{1)}\left(r, \frac{1}{G-1}\right) \\
& \leq N\left(r, \frac{1}{H}\right) \leq T(r, H)+O(1) \\
& \leq N(r, H)+S(r, f)+S(r, g) .
\end{aligned}
$$


Since $E_{3)}(1 ; F)=E_{3)}(1 ; G)$, the zeros of $F$ with multiplicities 1,2 and 3 are not poles of $H$. We denote by $N_{0}\left(\frac{1}{F^{\prime}}\right)$ the counting function of those zeros of $F^{\prime}$ but not of $F(F-1)$, (counting multiplicities). $N_{0}\left(r, \frac{1}{G^{\prime}}\right)$ can be defined in the same way. From (2.1) and (2.3) and the fact that $f(z)$ and $g(z)$ are two non-constant entire functions, we deduce that

$$
\begin{aligned}
N_{1)}\left(r, \frac{1}{F-1}\right) \leq & \bar{N}_{(2}\left(r, \frac{1}{F}\right)+\bar{N}_{(2}\left(r, \frac{1}{G}\right)+\bar{N}_{0}\left(\frac{1}{F^{\prime}}\right)+\bar{N}_{0}\left(\frac{1}{G^{\prime}}\right) \\
& +\bar{N}_{(4}\left(r, \frac{1}{F-1}\right)+\bar{N}_{(4}\left(r, \frac{1}{G-1}\right)+S(r, f)+S(r, g) .
\end{aligned}
$$

By the second fundamental theorem, we have

$$
\begin{aligned}
& T(r, F) \leq \bar{N}\left(r, \frac{1}{F}\right)+\bar{N}\left(r, \frac{1}{F-1}\right)-N_{0}\left(\frac{1}{F^{\prime}}\right)+S(r, F) . \\
& T(r, G) \leq \bar{N}\left(r, \frac{1}{G}\right)+\bar{N}\left(r, \frac{1}{G-1}\right)-N_{0}\left(\frac{1}{G^{\prime}}\right)+S(r, G) .
\end{aligned}
$$

Note that

$$
\begin{aligned}
& \bar{N}\left(r, \frac{1}{F-1}\right)-\frac{1}{2} \bar{N}_{1)}\left(r, \frac{1}{F-1}\right)+\bar{N}_{(4}\left(r, \frac{1}{F-1}\right) \\
\leq & \frac{1}{2} N\left(r, \frac{1}{F-1}\right) \leq \frac{1}{2} T(r, F)+O(1),
\end{aligned}
$$

and

$$
\begin{aligned}
& \bar{N}\left(r, \frac{1}{G-1}\right)-\frac{1}{2} \bar{N}_{1)}\left(r, \frac{1}{G-1}\right)+\bar{N}_{(4}\left(r, \frac{1}{G-1}\right) \\
\leq & \frac{1}{2} N\left(r, \frac{1}{G-1}\right) \leq \frac{1}{2} T(r, G)+O(1) .
\end{aligned}
$$

From $(2.3) \sim(2.8)$ we have

$$
\begin{aligned}
T(r, F)+T(r, G) & \leq 2\left(\bar{N}\left(r, \frac{1}{F}\right)+\bar{N}_{(2}\left(r, \frac{1}{F}\right)+\bar{N}\left(r, \frac{1}{G}\right)\right. \\
& \left.+\bar{N}_{(2}\left(r, \frac{1}{G}\right)\right)+S(r, f)+S(r, g) .
\end{aligned}
$$

Note that $T(r, F)=T\left(r, \frac{1}{F}\right)+S(r, f)$ and $T(r, G)=T\left(r, \frac{1}{G}\right)+S(r, g)$. It follows from (2.9) that

$$
\begin{aligned}
T\left(r, \frac{1}{F}\right)+T\left(r, \frac{1}{G}\right) \leq & 2\left(N\left(r, \frac{1}{F}\right)-\left[N_{(3}\left(r, \frac{1}{F}\right)-2 \bar{N}_{(3}\left(r, \frac{1}{F}\right)\right]+N\left(r, \frac{1}{G}\right)\right. \\
& \left.-\left[N_{(3}\left(r, \frac{1}{G}\right)-2 \bar{N}_{(3}\left(r, \frac{1}{G}\right)\right]\right)+S(r, f)+S(r, g) .
\end{aligned}
$$


We see that if $z_{0}$ is a zero of $f$ with multiplicity $l, l \geq 1$, then $z_{0}$ is a zero $F$ with multiplicity at least 3 since $n l-k>(k+2) l-k \geq 2$, so we get

$$
N_{(3}\left(r, \frac{1}{F}\right)-2 \bar{N}_{(3}\left(r, \frac{1}{F}\right) \geq(n-k-2) N\left(r, \frac{1}{f}\right)
$$

and

$$
N_{(3}\left(r, \frac{1}{G}\right)-2 \bar{N}_{(3}\left(r, \frac{1}{G}\right) \geq(n-k-2) N\left(r, \frac{1}{g}\right) .
$$

Note that $m\left(r, \frac{1}{F}\right)=T\left(r, \frac{1}{F}\right)-N\left(r, \frac{1}{F}\right)$ and $m\left(r, \frac{1}{G}\right)=T\left(r, \frac{1}{G}\right)-N\left(r, \frac{1}{G}\right)$. From (2.10), (2.11), (2.12) we have (2.2). This completes the proof of Lemma 2.4.

Lemma 2.5. Let $f(z)$ and $g(z)$ be two non-constant entire functions, let $n, m$, and $k$ be three positive integers with $n>k+2$ and $\lambda, \mu$ be constants such that $|\lambda|+|\mu| \neq 0$. Let $H$ be defined as (2.1). If $E_{2)}(1 ; F)=E_{2)}(1 ; G)$ and $H \not \equiv 0$, then

$$
\begin{aligned}
& m\left(r, \frac{1}{F}\right)+m\left(r, \frac{1}{G}\right) \leq N\left(r, \frac{1}{F}\right)+N\left(r, \frac{1}{G}\right)-2(n-k-2) N\left(r, \frac{1}{f}\right) \\
& -2(n-k-2) N\left(r, \frac{1}{g}\right)+\frac{1}{2} \bar{N}\left(r, \frac{1}{F}\right)+\frac{1}{2} \bar{N}\left(r, \frac{1}{G}\right)+S(r, f)+S(r, g) .
\end{aligned}
$$

Proof. Similar to the proof of Lemma 2.4, we have

$$
\begin{aligned}
N_{1)}\left(r, \frac{1}{F-1}\right) \leq & \bar{N}_{(2}\left(r, \frac{1}{F}\right)+\bar{N}_{(2}\left(r, \frac{1}{G}\right)+N_{0}\left(\frac{1}{F^{\prime}}\right)+N_{0}\left(\frac{1}{G^{\prime}}\right) \\
& +\bar{N}_{(3}\left(r, \frac{1}{F-1}\right)+\bar{N}_{(3}\left(r, \frac{1}{G-1}\right)+S(r, f)+S(r, g) .
\end{aligned}
$$

Note that

$$
\begin{aligned}
& \bar{N}\left(r, \frac{1}{F-1}\right)-\frac{1}{2} \bar{N}_{1)}\left(r, \frac{1}{F-1}\right)+\frac{1}{2} \bar{N}_{(3}\left(r, \frac{1}{F-1}\right) \\
\leq & \frac{1}{2} N\left(r, \frac{1}{F-1}\right) \leq \frac{1}{2} T(r, F)+O(1),
\end{aligned}
$$

and

$$
\begin{aligned}
& \bar{N}\left(r, \frac{1}{G-1}\right)-\frac{1}{2} \bar{N}_{1)}\left(r, \frac{1}{G-1}\right)+\frac{1}{2} \bar{N}_{(3}\left(r, \frac{1}{G-1}\right) \\
\leq & \frac{1}{2} N\left(r, \frac{1}{G-1}\right) \leq \frac{1}{2} T(r, G)+O(1) .
\end{aligned}
$$

From (2.5), (2.6) and (2.14) (2.16), we have

$$
\begin{aligned}
T(r, F)+T(r, G) \leq & 2\left(\bar{N}\left(r, \frac{1}{F}\right)+\bar{N}_{(2}\left(r, \frac{1}{F}\right)+\bar{N}\left(r, \frac{1}{G}\right)+\bar{N}_{(2}\left(r, \frac{1}{G}\right)\right) \\
& +\bar{N}_{(3}\left(r, \frac{1}{F-1}\right)+\bar{N}_{(3}\left(r, \frac{1}{G-1}\right)+S(r, f)+S(r, g) .
\end{aligned}
$$


As

$$
\bar{N}_{(3}\left(r, \frac{1}{F-1}\right) \leq \frac{1}{2} N\left(r, \frac{F}{F^{\prime}}\right) \leq \frac{1}{2} N\left(r, \frac{F^{\prime}}{F}\right)+S(r, f) \leq \frac{1}{2} \bar{N}\left(r, \frac{1}{F}\right)+S(r, f),
$$

similarly we have

$$
\bar{N}_{(3}\left(r, \frac{1}{G-1}\right) \leq \frac{1}{2} \bar{N}\left(r, \frac{1}{G}\right)+S(r, g) .
$$

Using the similar proof of Lemma 2.4 we get (2.13). This completes the proof of Lemma 2.5.

Lemma 2.6. Let $f(z)$ and $g(z)$ be two non-constant entire functions, let $n, m$, and $k$ be three positive integers with $n>k+2$ and $\lambda, \mu$ be constants such that $|\lambda|+|\mu| \neq 0$. Let $H$ be defined as (1). If $E_{1)}(1 ; F)=E_{1)}(1 ; G)$ and $H \not \equiv 0$, then

$$
\begin{aligned}
& m\left(r, \frac{1}{F}\right)+m\left(r, \frac{1}{G}\right) \leq N\left(r, \frac{1}{F}\right)+N\left(r, \frac{1}{G}\right)-2(n-k-2) N\left(r, \frac{1}{f}\right) \\
& -2(n-k-2) N\left(r, \frac{1}{g}\right)+2 \bar{N}\left(r, \frac{1}{F}\right)+2 \bar{N}\left(r, \frac{1}{G}\right)+S(r, f)+S(r, g) .
\end{aligned}
$$

Proof. Similar to the proof of Lemma 2.4, we have

$$
\begin{aligned}
N_{1)}\left(r, \frac{1}{F-1}\right) \leq & \bar{N}_{(2}\left(r, \frac{1}{F}\right)+\bar{N}_{(2}\left(r, \frac{1}{G}\right)+N_{0}\left(\frac{1}{F^{\prime}}\right)+N_{0}\left(\frac{1}{G^{\prime}}\right) \\
& +\bar{N}_{(2}\left(r, \frac{1}{F-1}\right)+\bar{N}_{(2}\left(r, \frac{1}{G-1}\right)+S(r, f)+S(r, g) .
\end{aligned}
$$

Note that

$$
\begin{aligned}
& \bar{N}\left(r, \frac{1}{F-1}\right)-\frac{1}{2} \bar{N}_{1)}\left(r, \frac{1}{F-1}\right) \\
\leq & \frac{1}{2} N\left(r, \frac{1}{F-1}\right) \leq \frac{1}{2} T(r, F)+O(1),
\end{aligned}
$$

and

$$
\begin{aligned}
& \bar{N}\left(r, \frac{1}{G-1}\right)-\frac{1}{2} \bar{N}_{1)}\left(r, \frac{1}{G-1}\right) \\
\leq & \frac{1}{2} N\left(r, \frac{1}{G-1}\right) \leq \frac{1}{2} T(r, G)+O(1) .
\end{aligned}
$$

From (2.5), (2.6) and (2.19) (2.21), we have

$$
\begin{aligned}
T(r, F)+T(r, G) & \leq 2\left(\bar{N}\left(r, \frac{1}{F}\right)+\bar{N}_{(2}\left(r, \frac{1}{F}\right)+\bar{N}\left(r, \frac{1}{G}\right)+\bar{N}_{(2}\left(r, \frac{1}{G}\right)\right. \\
& \left.+\bar{N}_{(2}\left(r, \frac{1}{F-1}\right)+\bar{N}_{(2}\left(r, \frac{1}{G-1}\right)\right)+S(r, f)+S(r, g) .
\end{aligned}
$$


Since

$$
\bar{N}_{(2}\left(r, \frac{1}{F-1}\right) \leq N\left(r, \frac{F}{F^{\prime}}\right) \leq N\left(r, \frac{F^{\prime}}{F}\right)+S(r, f) \leq \bar{N}\left(r, \frac{1}{F}\right)+S(r, f) .
$$

Similarly we have

$$
\bar{N}_{(2}\left(r, \frac{1}{G-1}\right) \leq \bar{N}\left(r, \frac{1}{G}\right)+S(r, g) .
$$

By the similar proof of Lemma 2.4 we get (2.18). This completes the proof of Lemma 2.6.

Remark 2.1. Suppose that the condition " $F=\left(f^{n}(z)\left(\mu f^{m}(z)+\lambda\right)\right)^{(k)}, G=$ $\left(g^{n}(z)\left(\mu g^{m}(z)+\lambda\right)\right)^{(k)}$ " is replaced by " $F=\left(f^{n}(z)(f(z)-1)^{m}\right)^{(k)}, G=$ $\left(g^{n}(z)(g(z)-1)^{m}\right)^{(k)}$ " in Lemmas 2.4-2.6, then the conclusions remain valid.

Lemma 2.7. Let $f(z)$ and $g(z)$ be two non-constant entire functions, let $n, m$ and $k$ be three positive integers with $n>2 k+m^{*}+4$, and $\lambda, \mu$ be constants such that $|\lambda|+|\mu| \neq 0$. Let $F=\left(f^{n}(z)\left(\mu f^{m}(z)+\lambda\right)\right)^{(k)}, G=\left(g^{n}(z)\left(\mu g^{m}(z)+\lambda\right)\right)^{(k)}, H$ be defined as (2.1). If $H \equiv 0$, then the conclusions of Theorem $\mathrm{F}$ hold.

Proof. As $H \equiv 0$, Proceeding as in the proof of Theorem 1 in [6], the conclusions of Theorem F hold, which also completes the proof of Lemma 2.7.

Lemma 2.8. Let $f$ and $g$ be two non-constant entire functions, and let $n, m, k$ be three positive integers with $n>2 k+m+4$. Let $F=\left(f^{n}(z)(f(z)-1)^{m}\right.$, $G=\left(g^{n}(z)(g(z)-1)^{m}\right)^{(k)}, H$ be defined as (1). If $H \equiv 0$, then the conclusions of the Theorem G hold.

Proof. As $H \equiv 0$, Proceeding as in the proof of Theorem 2 in [6], the conclusions of Theorem G hold, which also completes the proof of Lemma 2.8 .

\section{Proofs of the Theorems}

Proof of Theorem 1.1.

Suppose that $F, G, F^{*}, G^{*}$ and $H$ are defined as in Lemma 2.4. First by Lemma 2.1 we have

$$
\begin{aligned}
& T\left(r, F^{*}\right)=\left(n+m^{*}\right) T(r, f)+S(r, f) . \\
& T\left(r, G^{*}\right)=\left(n+m^{*}\right) T(r, g)+S(r, g) .
\end{aligned}
$$

Since $\left(F^{*}\right)^{(k)}=F$, we have

$$
m\left(r, \frac{1}{F^{*}}\right) \leq m\left(r, \frac{1}{F}\right)+S(r, f) .
$$

Note that 


$$
m\left(r, \frac{1}{F^{*}}\right)=T\left(r, \frac{1}{F^{*}}\right)-N\left(r, \frac{1}{F^{*}}\right) .
$$

Let $H$ be defined as (2.1). Suppose that $H \not \equiv 0$, by Lemma 2.4, we have

$$
\begin{aligned}
m\left(r, \frac{1}{F}\right)+m\left(r, \frac{1}{G}\right) \leq & N\left(r, \frac{1}{F}\right)+N\left(r, \frac{1}{G}\right)-2(n-k-2) N\left(r, \frac{1}{f}\right) \\
& -2(n-k-2) N\left(r, \frac{1}{g}\right)+S(r, f)+S(r, g) .
\end{aligned}
$$

Lemma 2.2 implies that

(3.6) $N\left(r, \frac{1}{F}\right) \leq N\left(r, \frac{1}{F^{*}}\right)+S(r, f), \quad N\left(r, \frac{1}{G}\right) \leq N\left(r, \frac{1}{G^{*}}\right)+S(r, g)$.

It follows From $(3.1) \sim(3.6)$ that

$$
\begin{aligned}
& \left(n+m^{*}\right)(T(r, f)+T(r, g)) \\
\leq \quad & N\left(r, \frac{1}{F^{*}}\right)-2(n-k-2) N\left(r, \frac{1}{f}\right)+N\left(r, \frac{1}{G^{*}}\right)-2(n-k-2) N\left(r, \frac{1}{g}\right) \\
& +N\left(r, \frac{1}{F}\right)+N\left(r, \frac{1}{G}\right)+S(r, f)+S(r, g) \\
\leq \quad & 2\left(N\left(r, \frac{1}{F^{*}}\right)+N\left(r, \frac{1}{G^{*}}\right)-(n-k-2) N\left(r, \frac{1}{f}\right)-(n-k-2) N\left(r, \frac{1}{g}\right)\right) \\
& +S(r, f)+S(r, g) \\
\leq \quad & 2\left[n N\left(r, \frac{1}{f}\right)+n N\left(r, \frac{1}{g}\right)+N\left(r, \frac{1}{\mu f^{m}+\lambda}\right)+N\left(r, \frac{1}{\mu g^{m}+\lambda}\right)\right. \\
& \left.-(n-k-2) N\left(r, \frac{1}{f}\right)-(n-k-2) N\left(r, \frac{1}{g}\right)\right]+S(r, f)+S(r, g) \\
\leq \quad & 2\left[N\left(r, \frac{1}{\mu f^{m}+\lambda}\right)+N\left(r, \frac{1}{\mu g^{m}+\lambda}\right)+(k+2)\left(N\left(r, \frac{1}{f}\right)+N\left(r, \frac{1}{g}\right)\right)\right] \\
& +S(r, f)+S(r, g) \\
\leq \quad & 2\left(m^{*}+k+2\right)(T(r, f)+T(r, g))+S(r, f)+S(r, g) .
\end{aligned}
$$

We obtain that $n \leq 2 k+m^{*}+4$, which contradicts $n>2 k+m^{*}+4$. Therefore, $H \equiv 0$. By Lemma 2.7, the conclusions of Theorem $\mathrm{F}$ hold. This completes the proof of Theorem 1.1.

Proof of Theorem 1.2.

Let $F, G, F^{*}, G^{*}$ and $H$ be defined as in Lemma 2.4. Suppose that $H \not \equiv 0$. By Lemma 2.5 we have

$$
\begin{array}{ll}
m\left(r, \frac{1}{F}\right)+m\left(r, \frac{1}{G}\right) \leq & N\left(r, \frac{1}{F}\right)+N\left(r, \frac{1}{G}\right)-2(n-k-2) N\left(r, \frac{1}{f}\right)-2(n-k \\
(3.7) & -2) N\left(r, \frac{1}{g}\right)+\frac{1}{2} \bar{N}\left(r, \frac{1}{F}\right)+\frac{1}{2} \bar{N}\left(r, \frac{1}{G}\right)+S(r, f)+S(r, g) .
\end{array}
$$


By Lemma 2.3 with $s=1$, together with $(3.1) \sim(3.4)$ and $(3.6)$, we get

$$
\begin{array}{ll} 
& \left(n+m^{*}\right)(T(r, f)+T(r, g)) \\
\leq \quad & N\left(r, \frac{1}{F^{*}}\right)-2(n-k-2) N\left(r, \frac{1}{f}\right)+N\left(r, \frac{1}{G^{*}}\right)-2(n-k-2) N\left(r, \frac{1}{g}\right) \\
& +N\left(r, \frac{1}{F}\right)+N\left(r, \frac{1}{G}\right)+\frac{1}{2} \bar{N}\left(r, \frac{1}{F}\right)+\frac{1}{2} \bar{N}\left(r, \frac{1}{G}\right)+S(r, f)+S(r, g) \\
\leq \quad & 2\left(N\left(r, \frac{1}{\mu f^{m}+\lambda}\right)+N\left(r, \frac{1}{\mu g^{m}+\lambda}\right)+(k+2)\left(N\left(r, \frac{1}{f}\right)+N\left(r, \frac{1}{g}\right)\right)\right) \\
& +\frac{1}{2}\left(\bar{N}\left(r, \frac{1}{\mu f^{m}+\lambda}\right)+\bar{N}\left(r, \frac{1}{\mu g^{m}+\lambda}\right)\right)+\frac{k+1}{2} \bar{N}\left(r, \frac{1}{f}\right) \\
& +\frac{k+1}{2} \bar{N}\left(r, \frac{1}{g}\right)+S(r, f)+S(r, g) \\
\leq \quad & {\left[2(k+2)+\frac{k+1}{2}+\frac{5 m^{*}}{2}\right](T(r, f)+T(r, g))+S(r, f)+S(r, g) .}
\end{array}
$$

We obtain that $n \leq \frac{5 k+3 m^{*}+9}{2}$, which contradicts with $n>\frac{5 k++3 m^{*}+9}{2}$. Hence $H \equiv 0$. By Lemma 2.7, the conclusions of Theorem $\mathrm{F}$ hold. This completes the proof of Theorem 1.2.

\section{Proof of Theorem 1.3.}

Let $F, G, F^{*}, G^{*}$ and $H$ be defined as in Lemma 2.4. Suppose that $H \not \equiv 0$. By Lemma 2.6, Lemma 2.3 with $s=1$, together with $(3.1) \sim(3.4)$ and (3.6), we get

$$
\begin{aligned}
& \left(n+m^{*}\right)(T(r, f)+T(r, g)) \\
\leq \quad & N\left(r, \frac{1}{F^{*}}\right)-2(n-k-2) N\left(r, \frac{1}{f}\right)+N\left(r, \frac{1}{G^{*}}\right)-2(n-k-2) N\left(r, \frac{1}{g}\right) \\
& +N\left(r, \frac{1}{F}\right)+N\left(r, \frac{1}{G}\right)+2 \bar{N}\left(r, \frac{1}{F}\right)+2 \bar{N}\left(r, \frac{1}{G}\right)+S(r, f)+S(r, g) \\
\leq \quad & 2\left(N\left(r, \frac{1}{F^{*}}\right)+N\left(r, \frac{1}{G^{*}}\right)-(n-k-2) N\left(r, \frac{1}{f}\right)-(n-k\right. \\
& \left.-2) N\left(r, \frac{1}{g}\right)\right)+2 N_{k+1}\left(r, \frac{1}{F^{*}}\right)+2 N_{k+1}\left(r, \frac{1}{G^{*}}\right)+S(r, f)+S(r, g) \\
\leq \quad & 2\left(n N\left(r, \frac{1}{f}\right)+n N\left(r, \frac{1}{g}\right)+N\left(r, \frac{1}{\mu f^{m}+\lambda}\right)+N\left(r, \frac{1}{\mu g^{m}+\lambda}\right)-(n-k\right. \\
& \left.-2) N\left(r, \frac{1}{f}\right)-(n-k-2) N\left(r, \frac{1}{g}\right)\right)+2(k+1) \bar{N}\left(r, \frac{1}{f}\right)+2(k+1) \bar{N}\left(r, \frac{1}{g}\right) \\
& +2\left(N\left(r, \frac{1}{\mu f^{m}+\lambda}\right)+N\left(r, \frac{1}{\mu g^{m}+\lambda}\right)\right)+S(r, f)+S(r, g) \\
\leq \quad & 2\left(2 N\left(r, \frac{1}{\mu f^{m}+\lambda}\right)+2 N\left(r, \frac{1}{\mu g^{m}+\lambda}\right)+(k+2)\left(N\left(r, \frac{1}{f}\right)+N\left(r, \frac{1}{g}\right)\right)\right) \\
& +2(k+1) \bar{N}\left(r, \frac{1}{f}\right)+2(k+1) \bar{N}\left(r, \frac{1}{g}\right)+S(r, f)+S(r, g) \\
\leq \quad & \left(4 k+4 m^{*}+6\right)(T(r, f)+T(r, g))+S(r, f)+S(r, g) .
\end{aligned}
$$


We obtain that $n \leq 4 k+3 m^{*}+6$, which contradicts $n>4 k+3 m^{*}+6$. Hence $H \equiv 0$. By Lemma 2.7, the conclusions of Theorem $\mathrm{F}$ hold. This completes the proof of Theorem 1.3.

Proof of Theorem 1.4.

Let

$$
\begin{aligned}
& \left.F=\left(f^{n}(z)(f(z)-1)^{m}\right)^{(k)}, \quad G=g^{n}(z)(g(z)-1)^{m}\right)^{(k)} . \\
& F^{*}=f^{n}(z)(f(z)-1)^{m}, \quad G^{*}=g^{n}(z)(g(z)-1)^{m} .
\end{aligned}
$$

Let $H$ be defined as in Lemma 2.5. By Lemma 2.1, we have

$$
\begin{aligned}
& T\left(r, F^{*}\right)=(n+m) T(r, f)+S(r, f) . \\
& T\left(r, G^{*}\right)=(n+m) T(r, g)+S(r, g) .
\end{aligned}
$$

Suppose that $H \not \equiv 0$. Proceeding as in the proof of Theorem 1.1, we can get a contradiction. Hence $H \equiv 0$. By Lemma 2.8, the conclusions of Theorem G hold. This completes the proof of Theorem 1.4.

Proof of Theorem 1.5.

Let

$$
\begin{aligned}
& \left.F=\left(f^{n}(z)(f(z)-1)^{m}\right)^{(k)}, \quad G=g^{n}(z)(g(z)-1)^{m}\right)^{(k)} . \\
& F^{*}=f^{n}(z)(f(z)-1)^{m}, \quad G^{*}=g^{n}(z)(g(z)-1)^{m} .
\end{aligned}
$$

Let $H$ be defined as in Lemma 2.4. Suppose that $H \not \equiv 0$. By Lemma 2.3 with $s=1$, we get

$$
\bar{N}\left(r, \frac{1}{F}\right) \leq N_{k+1}\left(r, \frac{1}{F^{*}}\right)+S(r, f)=N_{k+1}\left(r, \frac{1}{f^{n}(z)(f(z)-1)^{m}}\right)+S(r, f) .
$$

Since $n>k$, if $m \geq k+1,(3.10)$ implies

$$
\begin{aligned}
\bar{N}\left(r, \frac{1}{F}\right) & \leq(k+1) \bar{N}\left(r, \frac{1}{f}\right)+(k+1) \bar{N}\left(r, \frac{1}{f-1}\right)+S(r, f) \\
& \leq(k+1) \bar{N}\left(r, \frac{1}{f}\right)+(k+1) T(r, f)+S(r, f) .
\end{aligned}
$$

If $m<k+1,(3.10)$ implies

$$
\begin{aligned}
\bar{N}\left(r, \frac{1}{F}\right) & \leq(k+1) \bar{N}\left(r, \frac{1}{f}\right)+N\left(r, \frac{1}{(f-1)^{m}}\right)+S(r, f) \\
& \leq(k+1) \bar{N}\left(r, \frac{1}{f}\right)+m T(r, f)+S(r, f) .
\end{aligned}
$$

Combining (3.11) and (3.12) yields

$$
\bar{N}\left(r, \frac{1}{F}\right) \leq(k+1) \bar{N}\left(r, \frac{1}{f}\right)+\min \{m, k+1\} T(r, f)+S(r, f) .
$$


Similarly we have

$$
\bar{N}\left(r, \frac{1}{G}\right) \leq(k+1) \bar{N}\left(r, \frac{1}{g}\right)+\min \{m, k+1\} T(r, g)+S(r, g) .
$$

From (3.8), (3.9), (3.13) and (3.14), similar to the proof of Theorem 1.2, we get

$$
\begin{aligned}
& (n+m)(T(r, f)+T(r, g)) \\
\leq \quad & N\left(r, \frac{1}{F^{*}}\right)-2(n-k-2) N\left(r, \frac{1}{f}\right)+N\left(r, \frac{1}{G^{*}}\right)-2(n-k-2) N\left(r, \frac{1}{g}\right)+N\left(r, \frac{1}{F}\right) \\
& +N\left(r, \frac{1}{G}\right)+\frac{1}{2} \bar{N}\left(r, \frac{1}{F}\right)+\frac{1}{2} \bar{N}\left(r, \frac{1}{G}\right)+S(r, f)+S(r, g) \\
\leq & \frac{5 k+4 m+9+\min \{m, k+1\}}{2}(T(r, f)+T(r, g))+S(r, f)+S(r, g) .
\end{aligned}
$$

From this inequality above we obtain that $n \leq \frac{5 k+2 m+9+\min \{m, k+1\}}{2}$, which contradicts $n>\frac{5 k+2 m+9+\min \{m, k+1\}}{2}$. Hence $H \equiv 0$. By Lemma 2.8, the conclusions of Theorem G hold. This completes the proof of Theorem 1.5.

\section{Proof of Theorem 1.6.}

$$
\text { Let } \left.F=\left(f^{n}(z)(f(z)-1)^{m}\right)^{(k)}, \quad G=g^{n}(z)(g(z)-1)^{m}\right)^{(k)}, F^{*}=f^{n}(z)(f(z)-
$$
$1)^{m}, \quad G^{*}=g^{n}(z)(g(z)-1)^{m}$.

Let $H$ be defined as in Lemma 2.4. Suppose that $H \not \equiv 0$. Proceeding as in the proof of Theorem 1.2, we get

$$
\begin{aligned}
& (n+m)(T(r, f)+T(r, g)) \\
\leq \quad & N\left(r, \frac{1}{F^{*}}\right)-2(n-k-2) N\left(r, \frac{1}{f}\right)+N\left(r, \frac{1}{G^{*}}\right)-2(n-k-2) N\left(r, \frac{1}{g}\right)+N\left(r, \frac{1}{F}\right) \\
& +N\left(r, \frac{1}{G}\right)+2 \bar{N}\left(r, \frac{1}{F}\right)+2 \bar{N}\left(r, \frac{1}{G}\right)+S(r, f)+S(r, g) \\
\leq \quad & 2\left[N\left(r, \frac{1}{F^{*}}\right)+N\left(r, \frac{1}{G^{*}}\right)-(n-k-2) N\left(r, \frac{1}{f}\right)-(n-k-2) N\left(r, \frac{1}{g}\right)\right] \\
& +2 N_{k+1}\left(r, \frac{1}{F^{*}}\right)+2 N_{k+1}\left(r, \frac{1}{G^{*}}\right)+S(r, f)+S(r, g) \\
\leq \quad & 2\left[n N\left(r, \frac{1}{f}\right)+n N\left(r, \frac{1}{g}\right)+m N\left(r, \frac{1}{f-1}\right)+m N\left(r, \frac{1}{g-1}\right)-(n-k-2) N\left(r, \frac{1}{f}\right)\right. \\
& \left.-(n-k-2) N\left(r, \frac{1}{g}\right)\right]+2(k+1)\left[\bar{N}\left(r, \frac{1}{f}\right)+\bar{N}\left(r, \frac{1}{g}\right)\right] \\
& +2 \min \{m, k+1\}(T(r, f)+T(r, g))+S(r, f)+S(r, g) \\
\leq \quad & 2\left(m N\left(r, \frac{1}{f-1}\right)+m N\left(r, \frac{1}{g-1}\right)+(k+2)\left(N\left(r, \frac{1}{f}\right)+N\left(r, \frac{1}{g}\right)\right)\right. \\
& +2(k+1)\left[\bar{N}\left(r, \frac{1}{f}\right)+\bar{N}\left(r, \frac{1}{g}\right)\right]+2 \min \{m, k+1\}(T(r, f)+T(r, g)) \\
& +S(r, f)+S(r, g) \\
\leq & 4 k+2 m+6+2 \min \{m, k+1\}(T(r, f)+T(r, g))+S(r, f)+S(r, g) .
\end{aligned}
$$


From this inequality above we obtain that $n \leq 4 k+m+6+2 \min \{m, k+1\}$, which contradicts $n>4 k+m+6+2 \min \{m, k+1\}$. Hence $H \equiv 0$. By Lemma 2.8, the conclusions of Theorem $\mathrm{G}$ hold. This completes the proof of Theorem 1.6.

\section{Annex remarks}

In this section, we would like to point out a gap that appears in the proof of Theorem 4 of [1]. In [1, P. 1203], on the first line below formula (6.8), the authors said:

"Let $z_{1}$ be a zero of $f-1$ of order $p_{1}$, then $z_{1}$ is zero of $\left[f^{n}(f-1)\right]^{(k)}$ of order $p_{1}-k$. Therefore from (6.7), we obtain

$$
p_{1}-k=n q_{1}+q_{1}+k,
$$

since $z_{1}$ is a pole of $g$ of order $q_{1} "$.

A question arises:

Question: If $p_{1} \leq k$, then $z_{1}$ is not a zero of $\left[f^{n}(f-1)\right]^{(k)}$, and thus not a pole of $g$. How to deal with this case?

A similar gap can also be found in the proof of Case 1.2 of Theorem H. Actually, whether the case (i) of Theorem $\mathrm{H}$ holds for meromorphic functions is still an open problem. The first author [15, Theorems 1.2-1.3] gave a partial answer to it.

Acknowledgements. The authors would like to thank the referee for valuable suggestions. This research was supported by the National Natural Science Foundation of China (Grant No. 11171184) and the Fundamental Research Funds for the Central Universities (Grant No. 3122013k008).

\section{References}

[1] S. S. Bhoosnurmath, R. S. Dyavanal, Uniqueness and value-sharing of meromorphic functions, Computers and Mathematics with Applications, 53(2007), 1191-1205.

[2] J. F. Chen, X. Y. Zhang, W. C. Lin and S. J. Chen, Uniqueness of entire functions that share one value, Computers and Mathematics with Applications, 56(2008), 30003014.

[3] M. L. Fang, Uniqueness and value-sharing of entire functions, Computers and Mathematics with Applications, 44(2002), 828-831

[4] M. L. Fang and X. H. Hua, Entire functions that share one value, Journal of Nanjing University Mathematical Biquarterly, 13(1996), 44-48.

[5] W. K. Hayman, Picard values of meromorphic functions and their derivatives, Annals of Mathematics, 70(1959), 9-42.

[6] L. P. Liu, Uniqueness of meromorphic functions and differential polynomials, Computers and Mathematics with Applications, 56(2008), 3236-3245. 
[7] X. G. Qi and J. Dou Some Further Results on Entire Functions That Share FixedPoints, Kyungpook Math. J., 42(2009), 771-777.

[8] J. P. Wang, Uniqueness of linear differential polynomials that share the value 1 CM, Kyungpook Math. J., 42(2002), 443-455.

[9] S. M. Wang and Z. S. Gao, Meromorphic Functions Sharing a Small Function, Abstract and Applied Analysis, vol. 2007, Article ID 60718, 6 pages, 2007. doi: $10.1155 / 2007 / 60718$.

[10] C. C. Yang and H. X. Yi, Uniqueness Theory of Meromorphic Functions, Kluwer Academic Publishers, Dordrecht, The Netherlands, 2003.

[11] C. C. Yang and X. H. Hua, Uniqueness and value-sharing of meromorphic functions, Annales Academiœ Scientiarum Fennicœ Mathematica, 22(1997), 395-406.

[12] J. L. Zhang and L. Z. Yang, Some results related to a conjecture of R. Brück, Journal of Inequalities in Pure and Applied Mathematics, 8(1)(2007), Art. 18.

[13] X. Y. Zhang and W. C. Lin, Uniqueness and value-sharing of entire functions, Journal of Mathematical Analysis and Applications, 343(2008), 938-950.

[14] X. Y. Zhang and W. C. Lin, Corrigendum to "Uniqueness and value-sharing entire functions" [J. Math. Anal. Appl. 343 (2008), 938-950], J. Math. Anal. Appl., 352(2009), 971.

[15] X. B. Zhang and J. F. Xu, Uniqueness of meromorphic functions sharing a small function and its applications, Computers and Mathematics with Applications 61(2011) $722-730$. 